\title{
Comunidad Andina y la discusión sobre la seguridad, 1969-2019: \\ Tránsito histórico y perspectivas para la reflexión
}

\author{
Diego Pérez Enríquez*
}

\section{Resumen}

La Comunidad Andina (CAN) ha promovido durante 50 años la integración regional, centrándose en aspectos económicos y comerciales. Durante este tiempo se ha negado (salvo en los años posteriores al 11 de septiembre de 2001) a la discusión sobre temas de seguridad, a pesar de que sus países tienen muy vivas y complejas dinámicas. Desde tal perspectiva, el principal interés de este trabajo es caracterizar esta discusión, resaltando el rol de Estados Unidos que ha impulsado una agenda que copa la discusión pero que promueve sus propios intereses, y por otro, la dependencia de la trayectoria institucional que limita a estos países para considerar integrarse en este ámbito. Para ello se desarrolla una revisión histórica de los procesos nacionales, así como del entramado de seguridad regional; a partir de este se observan las influencias sobre las decisiones nacionales, así como sobre la estructura de la CAN. Esto permite revelar las fuerzas en juego y las limitaciones inevitables para incorporar los temas de seguridad en el marco de reflexión de la institucionalidad andina. Se concluye resaltando que la inexistencia de un nivel de coordinación entre estos países reduce las posibilidades para generar reflexiones y líneas de acción comunes para la subregión, en un momento en que las demandas son acuciantes.

Palabras clave: seguridad, instituciones, agenda, discusión, integración, fuerzas, Andes

\section{The Andean Community and the discussion on security, 1969-2019. Historical development and perspectives for reflection}

\section{Abstract}

The Andean Community (CAN) has promoted regional integration for 50 years, focusing on economic and commercial aspects. During this time it has refused (except in the years after September 11, 2001) to discuss security issues, despite the fact that its countries have

* Docente investigador de la Escuela de Seguridad y Defensa, Instituto de Altos Estudios Nacionales, Quito. «diego.perez@iaen.edu.ec`.

Recibido: 27 de abril de 2020 | Revisado: 24 de agosto de 2020 | Aceptado: 21 de septiembre de 2020. Para citar este artículo: Pérez Enríquez, Diego. "Comunidad Andina y la discusión sobre la seguridad, 1969-2019. Tránsito histórico y perspectivas para la reflexión”. Comentario Internacional 20 (2020): 121 145. doi: $10.32719 / 26312549.2020 .20 .1 .7$ 
very lively and complex dynamics. From this perspective, the main interest of this work is to characterize this discussion, highlighting the role of the United States to foster an agenda that monopolizes the discussion but that at the same time promotes its own interests, as well as the dependence of the institutional trajectory that limits these countries to consider integrating in this area. To this end, a historical review of national processes is made, as well as of the regional security framework. From this, the influences on national decisions, and on the structure of the CAN are observed. This makes it possible to reveal the forces at stake and the inevitable limitations to incorporate security issues into the reflection framework of the Andean institutional framework. This analysis concludes by highlighting that the lack of coordination between these countries reduces the possibilities for generating reflections and common lines of action for the subregion, at a time when the demands are pressing.

Keywords: security, institutions, agenda, discussion, integration, powers, Andes

\section{Introducción}

D

esde su creación en 1969, la Comunidad Andina (CAN) ha trabajado fundamentalmente en la configuración de condiciones favorables para la integración entre Bolivia, Colombia, Ecuador y Perú en los aspectos económicos y comerciales. Los objetivos detallados en el Acuerdo de Cartagena concentran el esfuerzo de la institucionalidad andina en procurar, según reza el artículo 2 de este instrumento, "el desarrollo equilibrado y armónico (que) debe conducir a una distribución equitativa de los beneficios derivados de la integración entre los Países Miembros de modo de reducir las diferencias existentes entre ellos".

Así se delimita el ámbito de trabajo del organismo, dejando explícitamente por fuera la discusión sobre los problemas de la seguridad, mismos que se incluyeron en su agenda bajo la forma de aportes muy puntuales en el escenario pos11 de septiembre de 2001. Tomando este punto de partida, este documento rastrea la discusión de la CAN sobre la seguridad, reflexiona sobre los elementos de la coyuntura global y regional que han provocado que esta se integre a su trabajo, y, propone algunos temas de urgente atención en cuanto a la seguridad para sus países miembros.

El principal interés de este trabajo es caracterizar la discusión sobre la seguridad para los países de la región, donde se resalta la existencia de una doble dinámica: por una parte, Estados Unidos (EE. UU.) que ha impulsa-

1. Comunidad Andina, Acuerdo de Integración Subregional Andino-Acuerdo de Cartagena, 26 de mayo de 1969. 
do una agenda hemisférica en la que se promueven sus intereses, y por otro, los países de la subregión que lidian con sus propios procesos y demandas de seguridad. Se señala la inexistencia de un nivel de coordinación entre estos países para generar reflexiones y líneas de acción comunes para la subregión.

Con tal intención se plantea una revisión histórica que inicia en los albores del siglo XX, analizando la manera cómo los intereses estadounidenses sobre la región crearon una agenda predominante de seguridad que, además, se institucionaliza e incide sobre las fuerzas armadas del continente. En los casos nacionales se observa que las visiones militares predominantes de nacionalismo, anticomunismo y responsabilidades estatales sobre el desarrollo se consolidan en una aproximación a la seguridad que varía según las preocupaciones predominantes como consecuencia de sus dinámicas internas. Los dos elementos trazan una línea de trayectoria que va a perdurar en el tiempo y que, además, se suma a la problemática de la débil cooperación subregional en seguridad.

El surgimiento de la CAN y su exclusivo enfoque en los asuntos comerciales de la integración se interpretan desde la perspectiva del Estado Burocrático-Autoritario (BA), ${ }^{2}$ procurando retratar cómo las dinámicas económicas y de seguridad se constituyen en dos andariveles aparentemente incomunicados. Tal análisis es todavía introductorio, no obstante, abre la puerta para análisis más detallados en lo posterior.

En definitiva, se plantea que las perspectivas subregionales sobre seguridad operaron con independencia del proceso de integración, tanto desde la formalidad institucional andina, como desde la práctica estatal.

El artículo inicia con una aproximación teórica que plantea la manera cómo el modelo BA planteado por O’Donnell para interpretar las relaciones político-económicas de las dictaduras de Chile, Argentina, Uruguay y Brasil presenta algunas entradas de reflexión válidas para ubicar la naturaleza de las decisiones de los gobiernos andinos en el momento de creación de la CAN en 1969. En segundo lugar, se analiza desde una perspectiva histórica el entramado político e institucional que se genera desde EE. UU. para la región, diseñando una estructura que impactará sobre la manera en que los gobiernos militares sudamericanos se aproximarán a la proble-

2. Guillermo O'Donnell, "Reflexiones sobre las tendencias de cambio del Estado burocrático-autoritario", Revista Mexicana de Sociología 39, n. 1 (1977): 9-59. 
mática de la seguridad. La tercera parte se preocupa por la manera en que los gobiernos de Los Andes dirigen la discusión sobre la seguridad desde sus propios procesos políticos y sociales nacionales. Finalmente se consideran las líneas de trayectoria histórica y geopolítica mencionadas para analizar la forma en que durante el siglo XXI la CAN -en tanto institución- y sus países -desde sus dinámicas nacionales- han canalizado la discusión sobre la seguridad y cómo esta prefigura un escenario que demanda revisitar las concepciones de seguridad vigentes.

Es importante señalar que este artículo no sigue una cronología, sino que busca explicar la seguridad andina y los procesos conexos al surgimiento y evolución de la CAN. Existen tres elementos centrales en las reflexiones que se desarrollan en las páginas siguientes: primero, el Estado es un actor central para el desarrollo interno, y los países de la región andina asumen tal pretensión, con variantes en su implementación, existiendo siempre una línea de tensión en torno a tal postulado; segundo, los Estados Unidos juegan un rol fundamental en la determinación de las prioridades de seguridad para la región, tanto al momento de establecer las amenazas (internas y externas), como al señalar las estrategias para atenderlas; tercero, como se argumenta posteriormente, la Comunidad Andina no surge de las preocupaciones regionales sobre la seguridad, pero circunstancias coyunturales la han acercado a la discusión en tal ámbito, no obstante el multilateralismo y la cooperación han sido siempre complejos en esta temática.

\section{Incipiente modelo burocrático-autoritario en los Andes}

Este artículo toma el institucionalismo histórico para trazar el sentido del desarrollo de la CAN y las posiciones subregionales sobre la seguridad, a partir de la creación de esta. El punto de partida esta en el planteamiento teórico de que los hechos no están desconectados de sus antecedentes, su secuencia incide en la forma y resultados que guían a las propias instituciones; dado que estas tienden a ser dependientes de la trayectoria, los orígenes de estas afectan su naturaleza. ${ }^{3}$

3. Paul Pierson, Politics in Time: History, Institutions, and Social Analysis, $1^{\text {ra }}$ Ed. (Princeton: Princeton University Press, 2004). 
Se toma como punto de partida el año 1969, en que se firma el Acuerdo de Cartagena, no obstante, con el fin de comprender las condiciones que existen en el surgimiento de la institución se pasan a considerar los antecedentes sociales y políticos que la configuran, tanto desde la perspectiva nacional como desde la hemisférica. Es importante remarcar que no se puede ubicar a la CAN como un fruto directo de los gobiernos militares que antecedieron a su concreción, pero tampoco se la puede comprender sin ellos. La firma del Acuerdo de Cartagena ocurre en un contexto que revela una trayectoria dependiente de la manera en que los postulados generales de los gobiernos militares se insertaron en las visiones de promoción de un proceso de integración subregional vinculado directamente con el proceso de industrialización por sustitución de importaciones, central para la agenda de desarrollo latinoamericana. ${ }^{4}$

Entonces, los gobiernos andinos alinearon sus expectativas integracionistas con los postulados cepalinos, como se observa en el primer artículo del Acuerdo de Cartagena. ${ }^{5}$ Es importante señalar, sin embargo, que tal convergencia de los países estaba matizada por las características propias de los procesos políticos que atravesaba cada país. En el momento de la firma del acuerdo, Bolivia vivía un interregno civil atado a la matriz militar que se había promovido desde 1966; Perú promovía una versión intensa de desarrollismo militar; Ecuador atravesaba por un periodo de inestabilidad política que conduciría a una dictadura civil en 1970 y una militar dos años más tarde; Colombia, a contramano, era el único país que atravesaba un momento de estabilidad política desde 1958. Por otra parte, Chile, signatario original del Acuerdo, entraba a la última fase de su periodo de estabilidad política, mientras que Venezuela, que se uniría en 1971, veía consolidado el pacto de Puntofijo (1958) que igualmente estabilizó su sistema político.

A las dinámicas de sus procesos políticos se sumaba la existencia de organizaciones insurgentes que amenazaban la estabilidad en varios de estos países, y, junto con los conflictos limítrofes clásicos de Bolivia, Perú y Ecuador, configuraban el escenario de seguridad predominante. La agenda de EE. UU. sobre la región implicó, adicionalmente, el desarrollo de actividades anticomunistas, lo que tensionó aún más las condiciones locales. Sien-

4. Germánico Salgado, El Grupo Andino de hoy: eslabón hacia la integración de Sudamérica (Quito: Universidad Andina Simón Bolívar / Corporación Editora Nacional, 1998).

5. Comunidad Andina. Acuerdo de Integración Subregional Andino - Acuerdo de Cartagena. 
do este el escenario de seguridad andino, la visión predominante giraba en torno a las acciones de grupos que con un amplio apoyo soviético podían desestabilizar la región, por lo que reforzarla supuso dos acciones: promover gobiernos que adopten la agenda de seguridad hemisférica y que tomen el modelo de desarrollo capitalista.

Desde una perspectiva liberal se puede comprender cómo la interdependencia económica entre Estados garantiza la paz y también es instrumental para el status quo. ${ }^{6}$ Desde tal punto de partida, favorecer el mayor número de intercambios entre los países de la región permitiría su desarrollo y, por otra parte, trasladar sus capacidades para atender los escenarios internos. Con esta premisa, se vuelve necesaria una referencia al modelo de Estado burocrático-autoritario (BA) propuesto por O'Donnell. Si bien originalmente remitía a los casos de Argentina, Brasil, Chile y Uruguay, destacaba que en ellos se había constituido un tipo de Estado en que a) el acceso a las posiciones de dirección del Estado se restringía a miembros de organizaciones complejas y burocratizadas, b) predominaba un sistema de exclusión y desactivación política de los sectores populares, c) al que se añadía uno de exclusión económica, d) las cuestiones políticas y sociales se asumían como problemas "técnicos" que competían solo a las esferas gobernantes, y, e) se han transformado los mecanismos de acumulación en un marco de extensa industrialización.?

La totalidad de estos elementos no se observan en los casos andinos, sin embargo, algunos resaltan la coincidencia de los postulados cepalinos con los de la doctrinad de seguridad nacional (DSN). Bajo tal premisa, estos países mantuvieron 1) restringidos mecanismos de acceso al poder (militares o políticos provenientes de un limitadísimo sistema), 2) exclusión política del sector popular y sus aliados (inevitable en las dictaduras, y sumamente restringido en los sistemas venezolano y colombiano), 3) profundas desigualdades económicas, 4) el surgimiento de tecnocracias que convivían con las visiones asistencialistas del Estado. Las transformaciones en los mecanismos de acumulación no son apreciables en estos países, pues predominaban estructuras estatistas consideradas centrales para el desarrollo, que convivían con una dinámica asistencialista-clientelar como meca-

6. Robert Gilpin, The Political Economy of International Relations (Princeton: Princeton University Press, 1987).

7. O'Donnell, "Reflexiones sobre las tendencias de cambio del Estado burocratico-autoritario". 
nismo de atención a los excluidos. Con estas consideraciones, se propone pensar en una incipiente forma de BA en los países de la región andina.

Al considerar la débil industrialización, especialmente en Bolivia y Ecuador cuyas economías en ese momento eran fundamentalmente agrarias, no se podía considerar un sector privado desarrollado y de la naturaleza de los casos analizados por O'Donnell. De hecho, estos países mantuvieron un extenso debate (y decisiones contrapuestas) sobre la pertinencia de nacionalizar ciertos sectores de la industria. En ese vaivén entraba en debate la delimitación de los sectores estratégicos para la seguridad del Estado, definición que había residido tradicionalmente en las FF.AA. Mantener la estabilidad de los sistemas políticos nacionales en este ámbito condujo a múltiples negociaciones que no siempre arrojaron resultados duraderos. Por otra parte, las expectativas del sector popular en torno a su inclusión económica quedaron entrampadas en mecanismos top-down de políticas públicas, ${ }^{8}$ que se enmarcan desde una perspectiva de intensa participación estatal (desarrollista o keynesiana), lo que derivó en la formación de vastas redes clientelares.

En un contexto en que diversos sectores políticos estaban excluidos o limitados en su participación, la promoción de tal inclusión por algunos líderes fortaleció el "populismo" como fenómeno de respuesta a tales condiciones. ${ }^{9}$ Con sus características nacionales y variaciones ideológicas, los casos de Velasco Alvarado (Perú), Barrientos (Bolivia), Velasco Ibarra (Ecuador), Pérez Jimenez y Larrazabal (Venezuela), o Rojas Pinilla (Colombia, una vez fuera del poder) reconectan la narrativa del individuo que puede proveer acceso a los beneficios del Estado por fuera de cualquier mediación institucional. Eventualmente las FF.AA. señalarán como perniciosa esta dinámica y procurarán restringir la participación de este tipo de líderes y promoviendo sistemas políticos cerrados.

No se puede considerar el BA por fuera del capitalismo, lo que supone la existencia de un sector privado con capacidad de agenda y mecanismos estatales que garanticen la propiedad privada. Estas premisas, además, implican libertad de agencia para el primero, con el fin de que procure aquello

8. Paul Sabatier, "Top-down and Bottom-up Approaches to Implementation Research: A Critical Analysis and Suggested Synthesis", Journal of Public Policy 6, n. ${ }^{\circ} 1$ (1986): 21-48.

9. Ernesto Laclau, La razón populista (Buenos Aires: Fondo de Cultura Económica, 2005). 
que permita su lucro. Las determinaciones del Acuerdo de Cartagena apuntaban justamente a que este tipo de configuración se fortalezca como consecuencia de la integración de los mercados regionales. Las dinámicas entre los actores nacionales y los escenarios de seguridad impusieron una naturaleza institucional sobre la estructura regional que se probará difícil de superar aún en la actualidad. El rol político de las FF.AA. se caracteriza adecuadamente en la actualización que O'Donnell realiza sobre el BA: "[...] suelen ser lo más nacional y menos capitalista de las instituciones estatales”. ${ }^{10}$

La normalización económica promovida por los sectores capitalistas entró rápidamente en conflicto con las perspectivas más estatistas que postulaban los gobiernos que resultaron del primer retorno democrático (Venezuela y Colombia) y más aún con aquellos que tenían un claro gen desarrollista (Perú, y Ecuador desde 1972) o en los que el vínculo con la promoción del desarrollo era inevitable por la trayectoria histórica de las instituciones (Bolivia, incluso en el régimen de Banzer desde 1971). A esto se suma el carácter primario exportador de todos estos países, lo que ponía siempre en primera línea el problema de la delimitación de los sectores estratégicos (petróleo, gas, minería).

\section{La seguridad y sus definiciones en la perspectiva continental}

La manera de comprender la seguridad varía con el tiempo, el lugar y la manera en que una sociedad se ha constituido. ${ }^{11}$ Por su parte, el institucionalismo histórico plantea que en el transcurso del tiempo, las instituciones circunscriben sus ámbitos de acción, establecen las regularidades bajo las que sucederán las relaciones en las que están inscritas, y los mecanismos de incidencia sobre los individuos, así como los de su propia reproducción, con el fin de subsistir y conectar pasado, presente y futuro. ${ }^{12}$ Asumiendo se observa la regularidad en la influencia estadounidense sobre la agenda de seguridad hemisférica desde el siglo XIX.

10. Guillermo O'Donnell, Catacumbas (Prometeo Libros Editorial, 2008), 82.

11. Andrea Cavalletti, La mitología de la seguridad. La ciudad biopolítica (Buenos Aires: Adriana Hidalgo editora, 2010).

12. Pierson, Politics in Time; Paul Pierson y Theda Skocpol, "El institucionalismo histórico en la ciencia política contemporánea", Revista Uruguaya de Ciencia Política 17 (2008): 7-38. 
En 1823, EE. UU. promulgó la Doctrina Monroe, que buscaba la protección del continente, utilizando los medios de este país para contener intentos europeos de reconquista, considerando que ello garantizaría seguridad para EE. UU. ${ }^{13}$ Más adelante la Enmienda Platt estableció condiciones para las relaciones EE. UU.-Cuba, convirtiéndola casi en un protectorado; luego, el bloqueo naval a Venezuela por parte de Alemania, Inglaterra e Italia (1902-1903), llevó al corolario Roosevelt, que planteaba la posibilidad de intervención estadounidense directa sobre la región. ${ }^{14}$

Bajo esas determinaciones, en los inicios del siglo XX, varios gobiernos de la región imbricaron sus intereses nacionales -por si mismo dispersos y con múltiples actores involucrados- con la agenda de seguridad estadounidense. Cuando los juegos de fuerzas internos desplazaban dicha agenda, se abrían las posibilidades para intervenciones militares estadounidenses, como ocurrió en Honduras, ${ }^{15}$ Cuba, República Dominicana, Haití16 y Colombia. ${ }^{17}$ Esta dinámica política tuvo como límite geográfico y de acción al istmo de Panamá, cuando esta región se separó de Colombia para formar un Estado independiente en 1903, bajo el auspicio de Roosevelt. Para este presidente existía un valor estratégico en el proyecto del canal interoceánico, lo que "demandaba que el Caribe se convierta en un lago americano". ${ }^{18}$

EE. UU. mantuvo alguna incidencia sobre la región en los años siguientes, pero con la Segunda Guerra Mundial en marcha, su diplomacia promovió acciones de alcance continental, enfatizando en la necesidad de reaccionar colectivamente ante eventuales acciones militares alemanas en la región. Tras la guerra, EE. UU. procuró "[...] extender su frontera de seguridad al conjunto del continente, trató de imponer a todos los países de la región una tutela militar análoga, mutatis mutandis, en sus objetivos a la (de) su glacis caribeño", ${ }^{19}$ con lo que se impulsó una institucionalidad con-

13. James Monroe, «Transcript of Monroe Doctrine (1823)», Our Documents, 2 de diciembre de 1823.

14. Theodore Roosevelt,"Transcript of Theodore Roosevelts Corollary to the Monroe Doctrine (1905)", Our document, 6 de diciembre de 1904.

15. Alison Acker, Honduras. The making of a Banana Republic (Toronto: Between the lines, 1988).

16. David Bogen, "The law of humanitarian intervention: united states policy in cuba (1898) and in the dominican republic (1965)", Harvard International Law Journal 7, n. 2 (1966): 296-315; John Blassingame, "The Press and American Intervention in Haiti and the Dominican Republic, 1904-1920", Caribbean Studies 9, n. 2 (julio de 1969): 27-43.

17. Juan Santiago Correa, "Colombia y la Doctrina Monroe: el caso del Ferrocarril de Panamá y las intervenciones estadounidenses en el Istmo", Memorias, 11, n. 22 (2014): 107-32.

18. Serge Ricard, "The Roosevelt Corollary", Presidential Studies Quarterly 36, n. 1 (2006): 20.

19. Alain Rouquié, El Estado militar en América Latina (México D.F.: Siglo XXI Editores, 1984), 134. 
tinental que reiteró su vocación pacífica y, especialmente, la garantía común frente a intervenciones externas.

Este proceso era coincidente con el esfuerzo global de contención al comunismo soviético, ${ }^{20}$ y comenzó a institucionalizarse en la región mediante la cooperación militar y política. Así, la Escuela de las Américas (1946) estandarizará las concepciones de seguridad, proveyendo entrenamiento de inteligencia y contrainsurgencia, especialmente durante los años 60 y $70 .{ }^{21}$ La Tabla 1 detalla algunos hitos en la estructura de seguridad continental.

\section{Tabla 1}

\section{Estructura de seguridad continental}

\begin{tabular}{|c|c|c|c|c|}
\hline Año & Reunión & Instrumento & Institución creada & Elementos \\
\hline 1940 & $\begin{array}{l}\text { Segunda Reunión de } \\
\text { Consulta de los Mi- } \\
\text { nistros de Relaciones } \\
\text { Exteriores }\end{array}$ & $\begin{array}{l}\text { Declaración XV, sobre } \\
\text { Asistencia Recíproca } \\
\text { y Cooperación para } \\
\text { la Defensa de las Na- } \\
\text { ciones Americanas }\end{array}$ & & $\begin{array}{l}\text { Planteamiento del } \\
\text { concepto de defensa } \\
\text { colectiva }\end{array}$ \\
\hline 1942 & $\begin{array}{l}\text { Tercera Reunión de } \\
\text { Consulta de los Mi- } \\
\text { nistros de Relaciones } \\
\text { Exteriores }\end{array}$ & & $\begin{array}{l}\text { Junta Interamericana } \\
\text { de Defensa }\end{array}$ & $\begin{array}{l}\text { Carácter permanen- } \\
\text { te y preparación para } \\
\text { defensa del continen- } \\
\text { te bajo coordinación } \\
\text { de EE. UU. }\end{array}$ \\
\hline 1945 & $\begin{array}{l}\text { Conferencia Intera- } \\
\text { mericana sobre Pro- } \\
\text { blemas de la Guerra y } \\
\text { de la Paz }\end{array}$ & $\begin{array}{l}\text { Asistencia Recíproca } \\
\text { y Solidaridad Ameri- } \\
\text { cana "Acta de Cha- } \\
\text { pultepec". }\end{array}$ & & $\begin{array}{l}\text { Seguridad colectiva } \\
\text { y reacción común de } \\
\text { los Estados signata- } \\
\text { rios. }\end{array}$ \\
\hline 1946 & $\begin{array}{l}\text { Ejército de los Esta- } \\
\text { dos Unidos. }\end{array}$ & & $\begin{array}{l}\text { Escuela de las Amé- } \\
\text { ricas }\end{array}$ & $\begin{array}{l}\text { Formación, doctrina, } \\
\text { y entrenamiento para } \\
\text { actividades antico- } \\
\text { munistas en América } \\
\text { Latina. }\end{array}$ \\
\hline 1947 & $\begin{array}{l}\text { Conferencia Inte- } \\
\text { ramericana para el } \\
\text { Mantenimiento de la } \\
\text { Pazy la Seguridad del } \\
\text { Continente. }\end{array}$ & $\begin{array}{l}\text { Tratado Interamerica- } \\
\text { no de Asistencia Recí- } \\
\text { proca (TIAR) }\end{array}$ & & $\begin{array}{l}\text { Fortalecimiento del } \\
\text { concepto de seguri- } \\
\text { dad colectiva. }\end{array}$ \\
\hline
\end{tabular}

20. George Kennan, "George Kennan's “Long Telegram”", Digital Archive International History Decalssified, 22 de febrero de 1946

21. David Marcus Lauderback, "The U.S. Army School of the Americas: Mission and Policy during the Cold War" (tesis doctoral, University of Texas at Austin, 2004). 


\begin{tabular}{|c|c|c|c|c|}
\hline 1948 & $\begin{array}{l}\text { IX Conferencia Inter- } \\
\text { nacional Americana }\end{array}$ & $\begin{array}{l}\text { Carta de la Organiza- } \\
\text { ción de los Estados } \\
\text { Americanos }\end{array}$ & $\begin{array}{ll}\text { Organización de Es- } \\
\text { tados } & \text { Americanos } \\
\text { (OEA) } & \end{array}$ & $\begin{array}{l}\text { Ratificación de pre- } \\
\text { vención ante interfe- } \\
\text { rencia de terceros en } \\
\text { la región. }\end{array}$ \\
\hline 1967 & & $\begin{array}{l}\text { Tratado para la Pros- } \\
\text { cripción de las Ar- } \\
\text { mas Nucleares en la } \\
\text { América Latina y el } \\
\text { Caribe (Tratado de } \\
\text { Tlatelolco) }\end{array}$ & $\begin{array}{l}\text { Organismo para la } \\
\text { Proscripción de las } \\
\text { Armas Nucleares en } \\
\text { la América Latina y el } \\
\text { Caribe (OPANAL) }\end{array}$ & $\begin{array}{l}\text { Declaratoria de La- } \\
\text { tinoamérica como } \\
\text { zona de paz y libre de } \\
\text { armas nucleares. }\end{array}$ \\
\hline
\end{tabular}

Fuente: Lauderback; Junta Interamericana de Defensa; OEA; Conferencia Interamericana sobre Problemas de la Guerra y de la Paz; Conferencia Interamericana para el Mantenimiento de la Paz y la Seguridad del Continente; McCoy; Bitencourt, Gallo Cruz. ${ }^{22}$

Elaboración: propia.

\section{Gobiernos andinos y las líneas guía de la discusión sobre la seguridad}

Al retornar el enfoque a los países de la región andina, igualmente se constata la influencia de la agenda de EE. UU. sobre las agendas nacionales. A partir de la creación de la Escuela de las Américas (1946) hasta la conformación de la CAN (1969), se constata gran inestabilidad política en los países de la región; las visitas de políticos a los cuarteles, o el acceso castrense a los palacios presidenciales eran hechos de la política cotidiana. ${ }^{23}$ En este periodo de 23 años, casi un tercio del tiempo, los países en cuestión tuvieron un gobierno militar (ver: Tabla 2).

22. Lauderback, "The U.S. Army School of the Americas: Mission and Policy during the Cold War". Organización de los Estados Americanos, Carta de la Organización de los Estados Americanos, 27 de febrero de 1967; Conferencia Interamericana sobre Problemas de la Guerra y de la Paz, Asistencia Recíproca y Solidaridad Americana- Acta de Chapultepec, 6 de marzo de 1945; Organización de Estados Americanos, Tratado Interamericano de Asistencia Reciproca, 2 de septiembre de 1947; Katherine McCoy, "Trained to Torture? The Human Rights Effects of Military Training at the School of the Americas", Latin American Perspectives 32, n. 6 (2005): 47-64; Luis Bitencourt, "Latin American Security: Emerging Challenges", en Challenges of the Global Century. Report of the Project on Globalization and National Security, ed. Stephen Flanagan, Ellen Frost, y Richard Kugler (Washington D.C.: National Defense University, 2001), 895-913; Selina Gallo Cruz, "Protest and public relations: The reinvention of the US Army School of the Americas", Interface: a journal for and about social movements 7, n. 1 (2015): 322-50.

23. Scott Mainwaring, "Transitions to Democracy and Democratic Consolidation: Theoretical and Comparative Issues", The Helen Kellogg Institute for International Relations, 1989; Alain Rouquié y Stephen Suffern, "Los militares en la política latinoamericana desde 1930", en Historia de América Latina. Política y Sociedad desde 1930, ed. Leslie Bethell (Barcelona: Crítica Grijalbo Mondadori, 1997), 281-341. 
Tabla 2

Gobiernos militares de facto en región andina (1946 - 1969)

\begin{tabular}{|c|c|c|c|}
\hline \multirow[t]{5}{*}{ Bolivia } & $1951-1952$ & Gral. Hugo Balivián Rojas & \multirow[t]{5}{*}{6} \\
\hline & $196-1965$ & Gral. René Barrientos Ortuño & \\
\hline & 1966 & $\begin{array}{l}\text { Gral. René Barrientos Ortuño y GraD. Alfredo } \\
\text { Ovando Candia (co-presidencia) }\end{array}$ & \\
\hline & 1966 & GraD. Alfredo Ovando Candia & \\
\hline & $1966-1969$ & Gral. René Barrientos Ortuño & \\
\hline \multirow[t]{2}{*}{ Colombia } & 1953-1957 & Tnte. Gral. Gustavo Rojas Pinilla & \multirow[t]{2}{*}{5} \\
\hline & $1957-1958$ & $\begin{array}{l}\text { Junta Militar de Gobierno: } \\
\text { Gral.Gabriel París Gordillo } \\
\text { Gral.Deogracias Fonseca Espinosa } \\
\text { Valm. Rubén Piedrahíta Arango } \\
\text { BGral.Rafael Navas Pardo } \\
\text { BGral.Luis Ordóñez Castillo } \\
\end{array}$ & \\
\hline Ecuador & $1963-1966$ & $\begin{array}{l}\text { Junta Militar: } \\
\text { Calm. Ramón Castro Jijón } \\
\text { Gral. Luis Cabrera Sevilla } \\
\text { Gral. Marcos Gándara Enríquez } \\
\text { Cnl. Guillermo Freile Posso }\end{array}$ & 3 \\
\hline \multirow[t]{4}{*}{ Perú } & $1948-1950$ & Gral. Manuel A. Odría Amoretti & \multirow{4}{*}{$\begin{array}{l}9 \\
\text { (considerando } \\
1969 \text { como } \\
\text { año de corte) }\end{array}$} \\
\hline & $1962-1963$ & $\begin{array}{l}\text { Junta Militar: } \\
\text { Gral. Ricardo Pérez Godoy } \\
\text { Tnte. Gra.. Pedro Vargas Prada } \\
\text { Valm. Juan Francisco Torres Matos. }\end{array}$ & \\
\hline & 1963 & Gral. Nicolás Lindley López & \\
\hline & 1968-(1975) & Gral. Juan Francisco Velasco Alvarado & \\
\hline \multirow[t]{4}{*}{ Venezuela } & $1948-1950$ & $\begin{array}{l}\text { Triunvirato Militar: } \\
\text { Gral. Carlos Román Delgado Chalbaud Gómez } \\
\text { GraD. Marcos Pérez Jiménez } \\
\text { GraD. Luis Llovera Páez }\end{array}$ & \multirow[t]{4}{*}{8} \\
\hline & $1952-1953$ & \multirow[t]{2}{*}{ GraD. Marcos Pérez Jiménez } & \\
\hline & 1953-1958 & & \\
\hline & 1958 & Valm. Wolfgang Enrique Larrazábal Ugueto & \\
\hline
\end{tabular}

Fuente: Rouquié y Suffern. ${ }^{24}$

Elaboración: propia.

24. Rouquié y Suffern, "Los militares en la política latinoamericana desde 1930"; Rouquié, El Estado militar en América Latina; Brian Loveman, For La Patria: Politics and the Armed Forces in Latin America (Wilmington, Del: Rowman \& Littlefield Publishers, 1999). 
Mientras estos países abordaron las cuestiones de la seguridad, sumaron paulatinamente a su agenda el problema de las condiciones para el desarrollo. Loveman señala que existió un rol militar en la conformación primigenia de los Estados latinoamericanos, mismo que permitió a estas instituciones trazar proyectos nacionales de integración, pero ante la inequidad predominante, muchos de estos gobiernos militares optaron por introducir reformas sociales que aportarían al desarrollo con una fuerte intervención estatal..$^{25}$ Entre la oficialidad, la inestabilidad era leída como una consecuencia de la competencia entre representantes de elites económicas y políticas que carecían de un proyecto de Estado.

La manera en que se abordó el problema, sin embargo, tiene pronunciados matices, incluso dentro de cada país: en Perú, Odría mantuvo un gobierno de estrechos vínculos con los intereses comerciales estadounidenses, ${ }^{26}$ pero la dictadura de Velasco Alvarado promovió una visión desarrollista que se profundizaría en los años $70 .{ }^{27}$

En Ecuador, "los militares apostaron por una opción reformista y anticomunista dando viabilidad al proyecto desarrollista del centro". ${ }^{28}$ Así, iniciaron la primera reforma agraria, lo que sembró la visión de otro tipo de acción del Estado que se retomaría en el siguiente gobierno militar.

En Bolivia, los gobiernos de Barrientos/Ovando continuarán nominalmente con las reformas introducidas por la revolución de 1952, pero las vaciará de contenidos para promover una agenda antisindicalista y conservadora; desde 1969, tras la muerte del primero, habrá un breve giro hacia la nacionalización. ${ }^{29}$

Colombia bajo Rojas Pinilla, en cambio, "mantuvo una alianza sumisa frente a los objetivos internacionales respaldados por los Estados Unidos, quien sirvió de garante para la consecución de recursos económicos y

25. Loveman, For La Patria: Politics and the Armed Forces in Latin America.

26. Felicitas López, El gobierno militar de Manuel A. Odría en Perú (1948-1956): un vistazo diplomático (México D.F.: Universidad Nacional Autónoma de México, 2017).

27. Germán Albuquerque, "No alineamiento, tercermundismo y seguridad en Perú: la política exterior del gobierno de Juan Velasco Alvarado (1968-1980)", América Latina Hoy 75, (2017): 149-66.

28. Patricio Moncayo, "El golpe militar de 1963 y el fin de un período excepcional de estabilidad política", en Transiciones y rupturas. El Ecuador en la segunda mitad del siglo XX (Quito: FLACSO-Ministerio de Cultura, 2010), 338.

29. Carlota Casalino, "Bolivia desde la Revolución Boliviana hasta el gobierno de Evo Morales", Investigaciones sociales 19, n. 35 (2015): 101-13; Rouquié y Suffern, "Los militares en la política latinoamericana desde 1930". 
armas necesarias para fortalecer al estado frente a las amenazas internas y externas". ${ }^{30}$

En Venezuela, tanto el Triunvirato Militar como el Pérezjimenismo que siguieron, se caracterizaron por la búsqueda de la "[...] transformación racional del medio físico y mejoramiento de las condiciones rurales, intelectuales y materiales de los habitantes del país". ${ }^{31} \mathrm{El}$ caso de Chile ingresa a contramano en esta reflexión por su extensa tradición democrática; el gobierno de Frei Montalva se alineo claramente a la visión cepalina, apostó a la integración como vía para promover el desarrollo, y su gobierno fue visto por EE. UU. como una viable alternativa democrática a la influencia cubana en la región. ${ }^{32}$

A pesar de la diferente intensidad con que estos gobiernos se aproximaron a los principios del desarrollismo, su lugar común está en que tomaron como principal responsabilidad la devolución del orden, el fortalecimiento del Estado, y la promoción del desarrollo. En ello resultó funcional la doctrina anticomunista estadounidense, pues convergió con la represión a los militantes que se oponen al gobierno, señalando su naturaleza desestabilizadora. En esta dinámica común para los países de la región, se sentó una línea de trayectoria cuya agenda de desarrollo coincide temporalmente con la que en 1969 da origen a la Comunidad Andina.

\section{Trayectoria y transformaciones en la seguridad andina}

La institucionalidad creada para lograr la integración comercial atravesó altibajos inevitables por las condiciones globales y regionales. Asumir la incorporación de Venezuela (1971) y el retiro de Chile (1976), exigió reconfigurar los mecanismos y cronogramas de integración, ${ }^{33}$ no obstante, la

30. Carlos Murgueitio, "Los gobiernos militares de Marcos Pérez Jiménez y Gustavo Rojas Pinilla: nacionalismo, anticomunismo y sus relaciones con los Estados Unidos (1953-1957)”, Historia y Espacio 1, n. ${ }^{\circ} 25$ (2005): 48.

31. Frank Rodriguez, "La noción de democracia en tiempos de la dictadura militar (1948-1958)", Presente y Pasado. Revista de Historia 17, n. 33 (2012): 136.

32. Felipe Rivera, “¡Ni capitalistas, ni comunistas! Una Revolución en Libertad en Guerra Fría”, en Eduardo Frei Montalva: Fe, política y cambio social, ed. David Vásquez y Felipe Rivera Polo (Santiago de Chile: Ediciones Biblioteca del Congreso Nacional de Chile, 2013), 125-56.

33. Salgado, El Grupo Andino de hoy: eslabón hacia la integración de Sudamérica. 
institucionalidad logró avances que en la actualidad se aprecian en el Sistema Andino de Integración, ${ }^{34}$ configurado por una compleja red de instancias y mecanismos que permiten que el proceso iniciado en 1969, mantenga una importante capacidad de trabajo en múltiples ámbitos que fortalecen la integración.

El modelo BA destacado en páginas previas, permite explicar procesos nacionales en los que la principal preocupación fue siempre la articulación de los sectores económicos con el sistema global, dejando en último plano el ideal democrático. Ya sea por el carácter dictatorial de los gobiernos que coinciden con el primer momento de la Comunidad Andina o, ya en la post-transición, por la decepción ante las incapacidades de la democracia para resolver los problemas de la cotidianidad, es importante anotar que el carácter que este tiene permanece en tales sistemas políticos. Operando como una especie de fuerza contradictoria a la naturaleza antidemocrática de los líderes, las FF.AA. en estos países sostuvieron la necesidad de que el Estado mantenga capacidades de intervención sobre la economía. Ello implicó concebir responsabilidades sobre el desarrollo, que complementaba las responsabilidades históricas y tradicionales sobre la seguridad.

Para la seguridad, las definiciones y estrategias se remitían a lo nacional o a lo hemisférico, y rara vez referían a lo vecinal. Un contexto de características y concepciones westfalianas hasta finales de los años noventa dificultaba la cooperación abierta en este ámbito. Se debe destacar, sin embargo, que el surgimiento de comisiones de vecindad y de mecanismos de cooperación bilateral, permitieron una articulación más clara para los países de la región, que eventualmente dieron paso a la conformación de un mecanismo denominado Comisión Binacional Fronteriza (COMBIFRON) que ha operado para las relaciones bilaterales entre Venezuela, Colombia, Ecuador, Perú, Bolivia. ${ }^{35}$ Es importante destacar que las reuniones que se

34. Comunidad Andina-Secretaría General, "Sistema Andino de Integración SAI", Comunidad Andina, accedido el 7 de marzo de 2020.

35. Fabian Novak y Sandra Namihas, Cooperación en seguridad entre el Perú y sus vecinos: Amenazas no tradicionales (Lima: Instituto de Estudios Internacionales (IDEI) Pontificia Universidad Católica del Perú/Konrad Adenauer Stiftung, 2014); Eduardo Pastrana y Rafael Castro, "Retos, socios estratégicos y escenarios para la política exterior colombiana durante el posconflicto", Agenda Internacional XXIV, n. 35 (2017): 97-118; Dolores Bermeo Lara y Nathalie Pabón Ayala, "Las relaciones de seguridad entre Colombia y Ecuador: una nueva construcción de confianza", RESDAL, 2008; David González y Emilmar Rodríguez, "El papel del Ejército Nacional de Colombia en la construcción de escenarios de integración regional: un nuevo paradigma de la seguridad y la defensa", Perspectivas en Inteligencia 10, n. ${ }^{\circ} 19$ (2018): 179-92. 
dan en este nivel tienen un objetivo de coordinación de acciones, no obstante, no trazan agendas de mediano o largo plazo para la articulación de una agenda regional de seguridad.

Las perspectivas regionales de seguridad no coincidieron con el nivel de desarrollo institucional ni la articulación señalada para el caso de la Comunidad Andina, que nunca intervino directamente sobre estos por las razones descritas previamente. El problema de la seguridad -que se imbricaba inevitablemente con el de la democracia- se debía resolver al interior de cada país, mientras que en el ámbito del desarrollo el aporte de la institucionalidad regional se ceñía al problema del desarrollo de un mercado común. El propio diseño de la institucionalidad le impedía más incidencia sobre la seguridad. La diversidad de aproximaciones sobre la seguridad en cada uno de sus miembros permite comprender el límite afrontado por la institución en su diseño original, como se recuenta a continuación, los procesos nacionales tuvieron características tan particulares que únicamente cabía buscar su coincidencia en lo práctico. Al mismo tiempo, la discusión sobre la seguridad estaba copada por la agenda hemisférica y por la nacional, dejando espacio solo para lo operativo en lo binacional.

Así planteado, se puede resumir la variedad de casos nacionales de la siguiente manera. En Chile, el gobierno de Pinochet trastocó la relación Estado-sociedad, alineó su política interna y externa con los postulados de la DSN, y generó gravísimos costos humanos. ${ }^{36}$ En Bolivia, bajo Banzer, se reformaron las instituciones económicas y sociales, y se reincorporó al país en la esfera estadounidense luego de un serio intento de acercamiento a la Unión Soviética. ${ }^{37}$

Perú con Velasco Alvarado (1968-1975) y Ecuador con Rodríguez Lara (1972-1976), adoptaron el modelo desarrollista, se incorporaron a los países no-alineados, y, trazaron líneas de cooperación con países de la esfera soviética. ${ }^{38}$ Eventualmente, los cambios en el mando de estas dictadu-

36. Maureen Hiebert y Pablo Policier, "Genocide in Chile?: An assessment", en State Violence and Genocide in Latin America: The Cold War Years, ed. Marcia Esparza, Henry R. Huttenbach, y Daniel Feierstein (London: Routledge, 2010), 64-80.

37. Casalino, "Bolivia desde la Revolución Boliviana hasta el gobierno de Evo Morales".

38. Juan Velit Granda, "Política exterior del Perú durante el gobierno militar", Agenda Internacional 2, n. 5 (1995): 51-62; Albuquerque, "No alineamiento, tercermundismo y seguridad en Perú: la política exterior del gobierno de Juan Velasco Alvarado (1968-1980)"; Edison Macías Núñez, General Guillermo Rodríguez Lara (Quito: Centro de Estudios Históricos del Ejército, 2017). 
ras (1975 y 1976) trajeron un realineamiento con EE. UU. y el inicio de sus transiciones democráticas. Por otra parte, el contencioso limítrofe entre estas naciones no se resolvió en 1998, tras las confrontaciones de 1981 y 1995, y la cooperación regional para su resolución. ${ }^{39}$

En Venezuela la agenda de seguridad incluía el control de algunas facciones insurgentes que operaban desde los años 60, a pesar de que aquello no constituía la preocupación central. En los 70, el país adoptó una estrategia de proyección internacional que implicó el establecimiento de relaciones diplomáticas y comerciales con Hungría, URSS, China, la defensa de los intereses latinoamericanos y la incorporación al movimiento no-alineado. ${ }^{40}$ A pesar de tensiones coyunturales, EE. UU. se mantuvo como su principal socio.

Las transiciones democráticas fueron negociadas en Ecuador (1979), Perú (1980) y Bolivia (1982), por lo que no alteraron las premisas de dirección del Estado, pero los nuevos regímenes se inauguraron con la crisis de la deuda externa (1982). Así, el modelo de desarrollo de intensa participación y aporte estatal, cultivado durante las dictaduras, tuvo que replantearse en medio de fuertes tensiones sociales.

Bajo estas condiciones, en Perú, Sendero Luminoso (SL) -una derivación del Partido Comunista existente desde 1969- fortaleció sus capacidades de acción e intensificó sus operaciones terroristas en todo el país. En una dinámica similar a la que se verá en el caso colombiano, SL cooperó con organizaciones de narcotráfico para financiarse. ${ }^{41}$ Junto al Movimiento Revolucionario Tupac Amaru (MRTA), estas organizaciones generaron un periodo de intensa violencia que terminaría en 2000. La cooperación de EE. UU. resultó decisiva para que el gobierno de Alberto Fujimori elimine esta amenaza. ${ }^{42}$

39. Adrián Bonilla, "Fuerza, conflicto y negociación. Proceso político de la relación entre Ecuador y Perú", en Ecuador-Perú. Horizontes de la negociación y el conflicto, ed. Adrián Bonilla (Quito: FLACSO Sede Ecuador, 1999), 13-30.

40. Anabel Melet, "Relaciones diplomáticas entre Estados Unidos y Venezuela en los dos períodos presidenciales de Carlos Andrés Pérez: Perspectiva de una visión comparada de relaciones diplomáticas con un liderazgo ejercido en dos tiempos", Anuario del Instituto de Derecho Comparado de la Universidad de Carabobo 32 (2009): 81-119; Luis Uharte Pozas, "Venezuela: del ajuste neoliberal a la promesa de "socialismo de siglo XXI'”, Historia Actual Online Primavera, n. ${ }^{\circ} 16$ (2008): 127-47.

41. James Levy, "Funding Terrorism: A Closer Look at Organized Crime Activity and Lethality", Political Science 6 (2011).

42. Rafał Wordliczek, "Foreign policy of the United States towards a region of low interest: case study of Peru", Americana e-journal of american studies in hungary XII, n. ${ }^{\circ} 2$ (2015); Center for International Poli- 
Colombia es el caso más relevante por sus particularidades e incidencia hemisférica: su conflicto interno es el punto focal de la relación entre EE. UU. y la región hasta la actualidad. En 1964, surgen las Fuerzas Armadas Revolucionarias de Colombia (FARC), de inspiración comunista, y hacia los años 70 sus operaciones convergerán con las de las organizaciones dedicadas al cultivo, procesamiento y tráfico de drogas, que tienen su principal mercado en EE. UU. ${ }^{43}$ Esto impulsó a que estos gobiernos diseñen una estrategia para contener sus operaciones, ${ }^{44} \mathrm{y}$, a partir de entonces la cooperación militar estadounidense ha crecido hasta ser la primera en el continente (418.1 millones de dólares en 2019). ${ }^{45}$ Extrañamente, la estabilidad del sistema político colombiano lo convierte en un socio valioso y seguro para operar sobre la región.

Así, la estrategia estadounidense sobre la región ha girado alrededor de las relaciones bilaterales: inicialmente para la contención de la expansión comunista, y al final de la Guerra Fría, para profundizar el combate al narcotráfico y terrorismo. ${ }^{46}$ Dado que Bolivia, Colombia, Ecuador y Perú son países productores o de tránsito de drogas ilícitas, en 1991 EE. UU. implementó exenciones arancelarias para la importación de productos nacionales a cambio de la coordinación con este país para la lucha contra la producción, procesamiento y tráfico de drogas (ATPDEA). Este instrumento perdió su validez en las primeras décadas del 2000, pues Bolivia fue suspendida en 2009 luego de un diferendo diplomático con EE. UU, Perú y Colombia, lo rescindieron luego de concretar sus tratados de libre comercio bilateral (2009 y 2012), y, Ecuador renunció en 2013.

A pesar de lo señalado, EE. UU. mantiene la preminencia en la agenda de seguridad andina. Tras los atentados del 11/09/2001 la CAN desarrollará una serie de instrumentos que revelan el deseo de trabajar sobre seguridad (ver: Tabla 3), no obstante, son declarativos y carecieron de acciones

cy, "Security Aid Pivot Table - Programs Peru", Security Assistance Monitor, 2020; Cynthia McClintock, "The United States and Peru in the 1990s: Cooperation with a critical caveat on democratic standards", Department of Political Science The George Washington University, junio de 2000.

43. Francisco Leal, Seguridad Nacional a la deriva. Del Frente Nacional a la Posguerra Fría., $1^{\text {ra }}$ ed. (México D.F.: Alfaomega Grupo Editor, 2002).

44, Saul Rodríguez, "Building civilian militarism: Colombia, internal war, and militarization in a mid-term perspective", Security Dialogue, 49, n. ${ }^{\circ} 1-2$ (2018): 109-22.

45. June S. Beittel, "Colombia: Background and U.S. Relations R43813". Congressional Research Service, 29 de noviembre de 2019.

46. Comunidad Andina-Secretaría General, Paz y Seguridad en la Región Andina (Lima: Comunidad Andina, 2006), 31 . 
conexas. En ese sentido, el único documento oficial de la Comunidad Andina sobre seguridad reconoce que se requiere "[...] impulsar la efectiva incorporación de la temática sobre la integración, en su sentido más amplio, en las agendas políticas nacionales [...]". ${ }^{47}$ No obstante, estas se encontraban copadas con la agenda hemisférica

Tabla 3

Instrumentos de CAN sobre seguridad

\begin{tabular}{|l|l|}
\hline \multicolumn{1}{|c|}{ Nombre del instrumento } & Fecha \\
\hline $\begin{array}{l}\text { SG/di } 419 \\
\text { Compromiso de Lima } \\
\text { Carta Andina para la Paz y la Seguridad } \\
\text { Limitación y control de los gastos destinados a la defensa externa }\end{array}$ & $17 / 06 / 2002$ \\
\hline $\begin{array}{l}\text { SG/di } 488 \\
\text { Elementos de información para la participación de los países andinos en los } \\
\text { trabajos de revisión de la Seguridad Hemisférica }\end{array}$ & $20 / 02 / 2003$ \\
\hline $\begin{array}{l}\text { SG/dt 199 } \\
\text { Elementos para una visión Andina de la Seguridad }\end{array}$ & $20 / 02 / 2003$ \\
\hline $\begin{array}{l}\text { SG/di } 511 \\
\text { Ayuda memoria } \\
\text { Acuerdos alcanzados durante la reunión del grupo de alto nivel en materia } \\
\text { de seguridad y fomento de la confianza de la comunidad andina }\end{array}$ & $19 / 05 / 2003$ \\
\hline $\begin{array}{l}\text { SG/dt 235 } \\
\text { Los países andinos frente a la conferencia especial de Seguridad Hemisférica }\end{array}$ & $18 / 09 / 2003$ \\
\hline $\begin{array}{l}\text { SG/di } 593 \\
\text { Informe del seminario/Taller subregional hacia la conformación de un espacio } \\
\text { de libertad, justicia y seguridad: los mecanismos de cooperación policial y } \\
\text { judicial en la unión europea y la comunidad andina. }\end{array}$ & $19 / 01 / 2004$ \\
\hline $\begin{array}{l}\text { DECISION 587 } \\
\text { Lineamientos de la Política de Seguridad Externa Común Andina }\end{array}$ & $10 / 07 / 2004$ \\
\hline $\begin{array}{l}\text { SG/dt 268 } \\
\text { La Red Andina de Seguridad } \\
\text { Propuesta sobre su Estructura y Funciones }\end{array}$ & $07 / 09 / 2004$ \\
\hline $\begin{array}{l}\text { SG/RE.PDMI/I/INFORME } \\
\text { Reunión de autoridades para analizar una propuesta de decisión sobre lucha } \\
\text { contra la minería ilegal en la Comunidad Andina. }\end{array}$ & $06 / 07 / 2012$ \\
\hline
\end{tabular}

Fuente: Comunidad Andina.

Elaboración: propia.

47. Comunidad Andina-Secretaría General, "Documentos Oficiales", Comunidad Andina, accedido el 1 de abril de 2020. 
A partir de 2008, se generó un esquema subregional de integración (Unasur) que buscó la generación de pensamiento estratégico propio. ${ }^{48} \mathrm{Se}$ pretendía la eventual convergencia de CAN y Mercosur en este, pero toda su estructura entró en suspenso desde 2018, nuevamente a merced de los cambios nacionales y globales. En este tránsito, sin embargo, la CAN incorporó dos temas relevantes desde la perspectiva amplia de seguridad: prevención de desastres y minería ilegal. ${ }^{49}$

\section{Conclusiones}

Este artículo ha procurado mostrar tres fuerzas en juego en la discusión sobre la seguridad en la región andina. La primera surge de las tensiones internas en los sistemas políticos de estos países, en los que la impronta militar incidió sobre la matriz decisional para la definición de la seguridad como consustancial al desarrollo. Así, se trazó una responsabilidad del Estado sobre este; con divergencias nacionales, pero subsiste la noción y en algunos casos una dependencia de la trayectoria institucional en este sentido. Con sistemas han sido predominantemente excluyentes y jerárquicos, aunque pobremente industrializados, se los caracterizó como BA incipientes. Ello supone un desafío actual en términos de democratización.

La segunda vincula la influencia estadounidense sobre la definición de las agendas y prioridades de seguridad. Con una matriz de incidencia que data del siglo XIX, asentada en una diversidad de instrumentos, instituciones, y prácticas, más eficientes líneas de cooperación, se ha trazado una trayectoria en la que su predominancia ha sido innegable, aún cuando los países de la región han intentado tomar alguna distancia. Esta agenda, sin embargo, tiene una visión restringida a los fenómenos clave para EE. UU. en cada coyuntura. La relación bilateral que se desarrolla alrededor de estos funciona articulando doctrina y acciones regionales, pero en función de estos intereses, sin introducir un nivel de reflexión estratégica para la región.

48. Centro de Estudios Estratégicos de Defensa, Consejo de Defensa Suramericano, y Unión de Naciones Suramericanas, Institucionalidad de la Defensa en Suramerica (Buenos Aires: CEED-CDS UNASUR, 2015).

49. Comunidad Andina-Secretaría General, "Minería Ilegal", Comunidad Andina, accedido el 1 de abril de 2020; "Prevención de Desastres", Comunidad Andina, accedido el 1 de abril de 2020. 
Finalmente, la integración andina se pensó históricamente desde una institucionalidad con estrictos fines comerciales. Si bien los proyectos desarrollistas nacionales conectaban seguridad y desarrollo, al diseñar el mecanismo de integración se los escindió; predominaron las perspectivas nacionalistas, así como las resultantes de los procesos políticos nacionales. Más allá de declaraciones diplomáticas muy específicas, la institucionalidad no ha atendido a la reflexión sobre la seguridad.

No es banal la consideración sobre incorporar la seguridad en la reflexión de la institucionalidad regional andina, pues existen necesidades particulares a la región que no se resuelven en las agendas bilaterales, y que, superan cada vez más a las determinaciones realizadas desde los ámbitos nacionales. En ese sentido, profundizar en la reflexión y el trabajo sobre la seguridad permitiría una innovadora entrada para el fortalecimiento de la integración andina; con ello se podría romper una línea de trayectoria institucional e iniciar un nuevo, y actual, ámbito de especialización.

\section{Bibliografía}

Acker, Alison. Honduras. The making of a Banana Republic. Toronto: Between the lines, 1988.

Albuquerque, Germán. "No alineamiento, tercermundismo y seguridad en Perú: la política exterior del gobierno de Juan Velasco Alvarado (1968-1980)". América Latina Hoy 75 (2017): 149-66. doi:10.14201/alh201775

Beittel, June. "Colombia: Background and U.S. Relations R43813". Congressional Research Service. 29 de noviembre de 2019. ‘https://bit.ly/2GZbinc〉.

Bermeo Lara, Dolores, y Nathalie Pabón Ayala. "Las relaciones de seguridad entre Colombia y Ecuador: una nueva construcción de confianza". Red de Seguridad y Defensa de América Latina. 2008. ‘https://bit.ly/3nBLWg6».

Bitencourt, Luis. "Latin American Security: Emerging Challenges". En Challenges of the Global Century. Report of the Project on Globalization and National Security, editado por Stephen Flanagan, Ellen Frost, y Richard Kugler, 895-913. Washington D.C.: National Defense University, 2001.

Blassingame, John. "The Press and American Intervention in Haiti and the Dominican Republic, 1904-1920". Caribbean Studies 9, n. 2 (1969): 27-43.

Bogen, David. "The law of humanitarian intervention: united states policy in cuba (1898) and in the dominican republic (1965)". Harvard International Law Journal 7, n. 2 (1966): 296-315.

Bonilla, Adrián. "Fuerza, conflicto y negociación. Proceso político de la relación entre Ecuador y Perú". En Ecuador - Perú. Horizontes de la negociación y el conflicto, editado por Adrián Bonilla, 13-30. Quito: FLACSO Sede Ecuador, 1999. 
Casalino Sen, Carlota. "Bolivia desde la Revolución Boliviana hasta el gobierno de Evo Morales". Investigaciones sociales 19, n. ${ }^{\circ} 35$ (2015): 101-13.

Cavalletti, Andrea. La mitología de la seguridad. La ciudad biopolítica. Buenos Aires: Adriana Hidalgo editora, 2010.

Center for International Policy. "Security Aid Pivot Table - Programs Peru". Security Assistance Monitor, 2020. https://bit.ly/34P2tor .

Centro de Estudios Estratégicos de Defensa, Consejo de Defensa Suramericano, y Unión de Naciones Suramericanas. Institucionalidad de la Defensa en Suramerica. Buenos Aires: CEED-CDS UNASUR, 2015.

Comunidad Andina - Secretaría General. "Documentos Oficiales". Comunidad Andina. Accedido 1 de abril de 2020. https://bit.ly/2Fsqfhu .

"Minería Ilegal". Comunidad Andina. Accedido 1 de abril de 2020. «https://bit. ly/3nJO6u3.

"Prevención de Desastres". Comunidad Andina. Accedido 1 de abril de 2020. 〈https://bit.ly/34OheI5desastres.〉.

—. "Sistema Andino de Integración - SAI". Comunidad Andina. Accedido el 7 de marzo de 2020. https://bit.ly/3jTcZBt .

- Paz y Seguridad en la Región Andina. Lima: Comunidad Andina, 2006.

Comunidad Andina. Acuerdo de Integración Subregional Andino - Acuerdo de Cartagena. 26 de mayo de 1969. https://bit.ly/2IhWO2t .

Conferencia Interamericana sobre Problemas de la Guerra y de la Paz. Asistencia Recíproca y Solidaridad Americana- Acta de Chapultepec. 6 de marzo de 1945. https://bit.ly/31GG2ZA>.

Correa, Juan Santiago. "Colombia y la Doctrina Monroe: el caso del Ferrocarril de Panamá y las intervenciones estadounidenses en el Istmo". Memorias 11, n. 22 (2014): 107-32. doi:10.14482/memor.22.5830.

Gallo Cruz, Selina. "Protest and public relations: The reinvention of the US Army School of the Americas". Interface: a journal for and about social movements 7 , n. ${ }^{\circ} 1$ (2015): 322-50.

Gilpin, Robert. The Political Economy of International Relations. Princeton: Princeton University Press, 1987.

González Cuenca, David, y Emilmar Rodríguez Caldera. "El papel del Ejército Nacional de Colombia en la construcción de escenarios de integración regional: un nuevo paradigma de la seguridad y la defensa". Perspectivas en Inteligencia 10, n. ${ }^{\circ} 19$ (2018): 179-92.

Hiebert, Maureen, y Pablo Policier. "Genocide in Chile?: An assessment". En State Violence and Genocide in Latin America: The Cold War Years, editado por Marcia Esparza, Henry R. Huttenbach, y Daniel Feierstein, 64-80. London: Routledge, 2010.

Junta Interamericana de Defensa. "El Sistema Interamericano de Defensa". Junta Interamericana de Defensa. 28 de agosto de 2012. https://bit.ly/3jS94oz〉. 
Kennan, George. "George Kennan's "Long Telegram”'”. Digital Archive International History Declassified. 22 de febrero de 1946. https://bit.ly/30YRUhD.

Laclau, Ernesto. La razón populista. Buenos Aires: Fondo de Cultura Económica, 2005. Lauderback, David Marcus. "The U.S. Army School of the Americas: Mission and Policy during the Cold War". Tesis doctoral, University of Texas at Austin, 2004. ‘https://bit.ly/2SPeSD6>.

Leal Buitrago, Francisco. Seguridad Nacional a la deriva. Del Frente Nacional a la Posguerra Fría. $1^{\text {ra }}$ Ed. México D.F.: Alfaomega Grupo Editor, 2002.

Levy, James. "Funding Terrorism: A Closer Look at Organized Crime Activity and Lethality". Political Science 6 (2011). https://bit.ly/3dkEn8K.

López Portillo, Felicitas. El gobierno militar de Manuel A. Odría en Perú (1948-1956): un vistazo diplomático. México D.F.: Universidad Nacional Autónoma de México, 2017.

Loveman, Brian. For La Patria: Politics and the Armed Forces in Latin America. Wilmington: Rowman \& Littlefield Publishers, 1999.

Macías Núñez, Edison. General Guillermo Rodríguez Lara. 34 vols. Quito: Centro de Estudios Históricos del Ejército, 2017. ‘https://bit.ly/2IgnnoM».

Mainwaring, Scott. "Transitions to Democracy and Democratic Consolidation: Theoretical and Comparative Issues". The Helen Kellogg Institute for International Relations, 1989. ‘https://bit.ly/375uXwK'.

McClintock, Cynthia. "The United States and Peru in the 1990s: Cooperation with a critical caveat on democratic standards" Department of Political Science The George Washington University. Junio de 2000.

McCoy, Katherine. "Trained to Torture? The Human Rights Effects of Military Training at the School of the Americas". Latin American Perspectives, 32, n. ${ }^{\circ} 6$ (2005): 47-64.

Melet, Anabel. "Relaciones diplomáticas entre Estados Unidos y Venezuela en los dos períodos presidenciales de Carlos Andrés Pérez: Perspectiva de una visión comparada de relaciones diplomáticas con un liderazgo ejercido en dos tiempos". Anuario del Instituto de Derecho Comparado de la Universidad de Carabobo 32 (2009): 81-119.

Moncayo, Patricio. "El golpe militar de 1963 y el fin de un período excepcional de estabilidad política". En Transiciones y rupturas. El Ecuador en la segunda mitad del siglo XX, 291-340. Quito: FLACSO - Ministerio de Cultura, 2010. https:// bit.ly/31xZ5F8;.

Monroe, James. «Transcript of Monroe Doctrine (1823)». Our Documents, 2 de diciembre de 1823. «https://bit.ly/3nLlnp1 .

Murgueitio, Carlos Alberto. "Los gobiernos militares de Marcos Pérez Jiménez y Gustavo Rojas Pinilla: nacionalismo, anticomunismo y sus relaciones con los Estados Unidos (1953 - 1957)". Historia y Espacio 1, n. ${ }^{\circ} 25$ (2005): 39-97.

Novak, Fabián, y Sandra Namihas. Cooperación en seguridad entre el Perú y sus vecinos: Amenazas no tradicionales. Lima: Instituto de Estudios Internacionales (IDEI) 
Pontificia Universidad Católica del Perú / Konrad Adenauer Stiftung, 2014. 〈https://bit.ly/36X63Q7).

O’Donnell, Guillermo. Catacumbas. Prometeo Libros Editorial, 2008.

- "Reflexiones sobre las tendencias de cambio del Estado burocratico-autoritario". Revista Mexicana de Sociología 39, n. 1 (1977): 9-59.

Organización de los Estados Americanos. Carta de la Organización de los Estados Americanos. 27 de febrero de 1967. https://bit.ly/2GVdAUH >.

Organización de Estados Americanos. Tratado Interamericano de Asistencia Recíproca. 2 de septiembre de 1947. https://bit.ly/2SOfHwg〉.

Pastrana, Eduardo, y Rafael Castro. "Retos, socios estratégicos y escenarios para la política exterior colombiana durante el posconflicto". Agenda Internacional XXIV, n. 35 (2017): 97-118. doi:10.18800/agenda.201701.006.

Pierson, Paul. Politics in Time: History, Institutions, and Social Analysis. $1^{\text {ra }}$ Ed. Princeton: Princeton University Press, 2004.

Pierson, Paul, y Theda Skocpol. "El institucionalismo histórico en la ciencia política contemporánea". Revista Uruguaya de Ciencia Política 17 (2008): 7-38.

Ricard, Serge. "The Roosevelt Corollary". Presidential Studies Quarterly 36, n. ${ }^{\circ} 1$ (2006): 17-26.

Rivera Polo, Felipe. "¡Ni capitalistas, ni comunistas! Una Revolución en Libertad en Guerra Fría”. En Eduardo Frei Montalva: Fe, política y cambio social, editado por David Vásquez y Felipe Rivera Polo, 125-56. Santiago de Chile: Ediciones Biblioteca del Congreso Nacional de Chile, 2013.

Rodriguez, Frank. "La noción de democracia en tiempos de la dictadura militar (19481958)". Presente y Pasado. Revista de Historia 17, n. 33 (2012): 129-44.

Rodríguez, Saul. "Building civilian militarism: Colombia, internal war, and militarization in a mid-term perspective". Security Dialogue 49, n. ${ }^{\circ}$ 1-2 (2018): 109-22. doi:10.1177/0967010617743201.

Roosevelt, Theodore. "Transcript of Theodore Roosevelts Corollary to the Monroe Doctrine (1905)". Our documents. 6 de diciembre de 1904. https://bit. $1 \mathrm{y} / 3 \mathrm{k} 66 \mathrm{pYx}$.

Rouquié, Alain. El Estado militar en América Latina. México D.F.: Siglo XXI Editores, 1984.

Rouquié, Alain, y Stephen Suffern. "Los militares en la política latinoamericana desde 1930". En Historia de América Latina. Política y Sociedad desde 1930, editado por Leslie Bethell, 281-341. Barcelona: Crítica Grijalbo Mondadori, 1997.

Sabatier, Paul. "Top-down and Bottom-up Approaches to Implementation Research: A Critical Analysis and Suggested Synthesis". Journal of Public Policy 6, n. ${ }^{\circ} 1$ (1986): 21-48.

Salgado, Germánico. El Grupo Andino de hoy: eslabón hacia la integración de Sudamérica. Quito: Universidad Andina Simón Bolívar / Corporación Editora Nacional, 1998. 
Uharte Pozas, Luis. "Venezuela: del ajuste neoliberal a la promesa de 'socialismo de siglo XXI". Historia Actual Online Primavera, n. 16 (2008): 127-47.

Velit Granda, Juan. "Política exterior del Perú durante el gobierno militar". Agenda Internacional 2, n. ${ }^{\circ} 5$ (1995): 51-62.

Wordliczek, Rafał. "Foreign policy of the United States towards a region of low interest: case study of Peru". Americana e-journal of american studies in hungary XII, n. 2 (2015), ‘https://bit.ly/371xLLs`. 